\title{
Canadian
} Science Publishing

Canadian Journal of Physics

Revue canadienne de physique

\section{High-fidelity generating multi-qubit $W$ state via dressed states in the system of multiple resonators coupled with a superconducting qubit}

\begin{tabular}{|c|c|}
\hline Journal: & Canadian Journal of Physics \\
\hline Manuscript ID & cjp-2017-0227.R1 \\
\hline Manuscript Type: & Article \\
\hline Date Submitted by the Author: & 25-Jun-2017 \\
\hline Complete List of Authors: & $\begin{array}{l}\text { Yang, Yu-Feng; Quantum Informaiton, Physics } \\
\text { Chen, Ye-Hong; Quantum Informaiton, Physics } \\
\text { Wu, Qi-Cheng; Quantum Informaiton, Physics } \\
\text { Shi, Zhi-Cheng; Quantum Informaiton, Physics } \\
\text { Song, Jie; Harbin Institute of Technology } \\
\text { Xia, Yan; Quantum Informaiton, Physics }\end{array}$ \\
\hline Keyword: & $\begin{array}{l}\text { W state, Circuit quantum electrodynamics, Dressed state, Quantum Zeno } \\
\text { dynamics, Accelerating the adiabatic passage }\end{array}$ \\
\hline $\begin{array}{r}\text { Is the invited manuscript for } \\
\text { consideration in a Special } \\
\text { Issue? }\end{array}$ & N/A \\
\hline
\end{tabular}

SCHOLARONE

Manuscripts 


\title{
High-fidelity generating multi-qubit $W$ state via dressed states in the system of multiple resonators coupled with a superconducting qubit
}

\author{
Yu-Feng Yang ${ }^{1,2}$, Ye-Hong Chen ${ }^{1,2}$, Qi-Cheng $\mathrm{Wu}^{1,2}$, \\ Zhi-Cheng Shi ${ }^{1,2, *}$, Jie Song ${ }^{3}$, and Yan Xia ${ }^{1,2, \dagger}$ \\ ${ }^{1}$ Department of Physics, Fuzhou University, Fuzhou 350116, China \\ ${ }^{2}$ Fujian Key Laboratory of Quantum Information and \\ Quantum Optics (Fuzhou University), Fuzhou 350116, China \\ ${ }^{3}$ Department of Physics, Harbin Institute of Technology, Harbin 150001, China \\ In this paper, we present an alternative scheme to generate $W$ state of three \\ superconducting qubits in three spatially separated coplanar waveguide resonators \\ with the quantum Zeno dynamics and the dressed states. When a set of dressed states \\ is suitably chosen, a scheme can be designed for accelerating the adiabatic passage \\ without additional couplings. What's more, the populations of the intermediate \\ states of the system can be restricted with choosing suitable control parameters. \\ We discuss the influence of dissipation and operational imperfection by numerical \\ analysis and the results show that the scheme is robust against various decoherence \\ processes. In addition, we hope the scheme can provide a theoretical basis for the \\ manipulation of the multi-qubit quantum state.
}

PACS numbers: 03.67. Pp, 03.67. Mn, 03.67. HK

Keywords: $W$ state; Circuit quantum electrodynamics; Dressed state

\section{INTRODUCTION}

In recent years, much attention has been focused on production and manipulation of entangled states, which promoted the development of experimental technique and led to the realization of entangled states in a variety of physical systems [1-4]. As an important

\footnotetext{
* E-mail: szc2014@yeah.net

$\dagger$ E-mail: xia-208@163.com
} 
platform of quantum information processing (QIP), circuit cavity quantum electrodynamics (QED) system [5-13] is widely investigated. Recent studies show that the solid state systems [14-18] associated with circuit QED not only have high feasibility, but also have better controllability and enormous superiority. For example, long coherence time is available for implementing the quantum operations and the system could be easily scaled up and controlled. So, there have been numerous protocols [19-27] which were proposed to prepare a variety of quantum states based on circuit QED. Such as, Frederick et al. presented a method of generating entangled quantum superpositions of Fock states by two resonators coupling to a superconducting qubit [26]. Yang et al. investigated the generation of entangled coherent states through the coupling between four microwave resonators and a three-level superconducting device, and they created a Greenberger-Horne-Zeilinger (GHZ) state with four different resonators embedding four superconducting qubits, respectively [27]. As is known, GHZ states [28] and $W$ states [29] are multi-qubit entangled states which cannot be converted to each other under local operations assisted by classical communication. Different from GHZ states, the entanglement of $W$ states is not only more persistent and robust against the loss of qubit loss and bit flip noise, but also immune against global dephasing. Considering these interesting features of $W$ states, several approaches are developed to generate $W$ states [30-33]. For example, Wei and Chen utilized the circuit QED to effectively generate the $W$ state with Resonant interaction [30] and adiabatic passage [31], respectively.

In general, the resonant pulses and adiabatic passage techniques are two popular methods for generating $W$ states, each method has its benefits and disadvantages. For example, the scheme with resonant pulses is fast but very sensitive to parameter fluctuations, the adiabatic passage techniques is inherently more robust against pulse area and timing errors but is time consuming which will lead to dissipation. So, as to achieve the goal of fastness and robustness, the approaches of "Shortcuts to adiabatic passage" (STAP) [34-67] have been proposed. Among these approaches [34-55], transitionless driving [36], inverse engineering based on invariants [42], the method to accelerate the adiabatic passages by using dressed states [45] are some common used methods. Especially, the method to accelerate the adiabatic passages by using dressed states proposed by Baksic et al. [45] has attracted many researchers attention. In the method, by incorporating corrections to the initial control Hamiltonian, a perfect state transfer can be permitted even in the nonadiabatic regime. Besides, by suitably selecting control parameters, the population of the intermediate state 
can be well inhibited and the desired population transfer can be efficiently improved.

Motivated by the reference [45], here we come up with an efficient scheme to generate a $W$ state. In the scheme, based on the quantum Zeno dynamics (QZD) and the dressed states, this scheme has the following advantages: (i) two experimentally feasible pulses are designed to realize the generation of the $W$ state; (ii) The intermediate states are slightly populated; (iii) Numerical simulation demonstrates that the scheme is fast and robust against the decoherence caused by the decay of the cavity mode, the energy relaxation and the dephasing. Moreover, the scheme is quite insensitive to the variation of parameters.

The remainder of the paper is structured as follows. In Sec. II, we briefly review the QZD and describe the method to accelerate the adiabatic passages by using dressed states. In Sec. III, we propose a scheme to generate $W$ state by using dressed states in the circuit cavity QED. In Sec. IV, we make a numerical simulation and then analyze the feasibility in the experiment. Finally, Sec. V summarizes the main conclusions of this work.

\section{PRELIMINARY THEORY}

\section{A. Quantum Zeno dynamics}

For the sake of clearness, we first simply review the main ideas of quantum Zeno dynamics, which was raised by Facchi and Pascazio in 2002 [68]. Generally, supposing the system's Hamiltonian is described as $H_{K}=H_{o b s}+K H_{\text {meas }}$, where $H_{o b s}$ is the Hamiltonian of the investigated quantum system, $H_{\text {meas }}$ is an additional interaction Hamiltonian performing the measurement, $K$ is a coupling constant. In the limits $K \rightarrow \infty$, the effective Hamiltonian of the system is approximated as $H_{K}=\sum_{n^{\prime}}\left(K \eta_{n^{\prime}} P_{n^{\prime}}+P_{n^{\prime}} H_{o b s} P_{n^{\prime}}\right)$, where $P_{n^{\prime}}$ is the eigenprojections of $H_{\text {meas }}$ corresponding eigenvalues $\eta_{n^{\prime}}\left(H_{\text {meas }}=\sum_{n^{\prime}} \eta_{n^{\prime}} P_{n^{\prime}}\right)$. More interesting, the system will evolve away from its initial state, while it still remains in the so-called Zeno subspace determined by the measurement.

\section{B. The method to accelerate the adiabatic passage by using dressed states}

To start with, a unitary operator is defined as $U(t)=\sum_{n}\left|\varphi_{n}(t)\right\rangle\langle n|$, in which, the instantaneous eigenstates $\left\{\left|\varphi_{n}(t)\right\rangle\right\}$ of the original Hamiltonian $H_{0}(t)$ whose eigenvalues are $\left\{E_{n}(t)\right\}$, 
and $\{|n\rangle\}$ are a set of bare states of the system. In adiabatic frame, the Hamiltonian can be expressed as

$$
H_{a d}(t)=U^{\dagger}(t) H_{0}(t) U(t)+\Upsilon(t)=\sum_{n} E_{n}(t)|n\rangle\langle n|-i U^{\dagger}(t) \dot{U}(t)
$$

where $\Upsilon(t)=-i U^{\dagger}(t) \dot{U}(t)$ usually involves the coupling transition between the instantaneous eigenstates of $H_{0}(t)$ and leads nonadiabatic errors. To correct the nonadiabatic errors, a correction Hamiltonian $H_{c o}(t)$ is introduced. Then, the modified Hamiltonian is described as $H^{\prime}(t)=H_{0}(t)+H_{c o}(t)$. In adiabatic frame, the modified Hamiltonian is expressed by

$$
\begin{aligned}
H_{a d}^{\prime}(t) & =U^{\dagger}(t) H_{0}(t) U(t)+U^{\dagger}(t) H_{c o}(t) U(t)+\Upsilon(t) \\
& =\sum_{n} E_{n}(t)|n\rangle\langle n|+U^{\dagger}(t) H_{c o}(t) U(t)-i U^{\dagger}(t) \dot{U}(t) \\
& =H_{a d}(t)+U^{\dagger}(t) H_{c o}(t) U(t) .
\end{aligned}
$$

Next, another frame is defined by $V(t)=\sum_{n}\left|\tilde{\varphi}_{n}(t)\right\rangle\langle n|$, in which $\left\{\left|\tilde{\varphi}_{n}(t)\right\rangle\right\}$ is the suitable choice of dressed states. Supposing that the initial time is $t_{i}\left(t_{i}=0\right)$, while the final time is $t_{f}$ (the interaction time $T=t_{f}$ ), the unitary operator $V(t)$ meet the condition $V(0)=V(T)=1$. So, $H_{a d}^{\prime}(t)$ in adiabatic frame can be described

$$
H_{V}^{\prime}(t)=V^{\dagger}(t) H_{a d}(t) V(t)+V^{\dagger}(t) U^{\dagger}(t) H_{c o}(t) U(t) V(t)-i V^{\dagger}(t) \dot{V}(t)
$$

If we can design a suitable $H_{c o}(t)$ to meet the condition $\left\langle\tilde{\varphi}_{m^{\prime}}(t)\left|H_{V}^{\prime}(t)\right| \tilde{\varphi}_{n}(t)\right\rangle=0\left(m^{\prime} \neq n\right)$, the unwanted off-diagonal elements in $H_{V}(t)$ can be cancelled. So, the shortcuts to adiabatic passage can be constructed.

\section{GENERATION OF THREE-QUBIT $W$ STATE VIA DRESSED STATES}

In this section, we introduce the generation of a $W$ state via dressed states in detail. We consider that a system involves a superconducting coupler (SCC) qubit, three spatially separated coplanar waveguide resonators $\left(C P W R_{1}, C P W R_{2}, C P W R_{3}\right)$ and three superconducting qubits $\left(S Q_{1}, S Q_{2}, S Q_{3}\right)$. The schematic diagram is shown in Fig. 1 (a), the $C P W R_{m}(m=1,2,3)$ is coupled to a single SCC qubit placed in the center of the devices by capacitor $C_{m}$. The $S Q_{m}$ places in the antinodes of the $C P W R_{m}$, respectively. As shown 
in Fig. 1 (b), the $S Q_{m}$ has an excited state $|e\rangle_{m}(\mathrm{~m}=1,2,3)$ and two ground states $|f\rangle_{m}$ and $|g\rangle_{m}$. The transition $|e\rangle_{m} \leftrightarrow|f\rangle_{m}$ is driven resonantly by an external classical field with Rabi frequency $\Omega_{m}(t)$ and the transitions $|e\rangle_{m} \leftrightarrow|g\rangle_{m}$ is resonantly coupled to the corresponding $C P W R_{m}$ with coupling strengths $v_{m}$. The SCC qubit has an excited state $|e\rangle_{c}$ and two ground states $|f\rangle_{c}$ and $|g\rangle_{c}$, it's structure is similar to the $S Q_{m}$. Different from the $S Q_{m}$ the transition $|e\rangle_{c} \leftrightarrow|g\rangle_{c}$ is resonantly coupled to the $C P W R_{m}$ with coupling strength $\lambda_{m}$. While the transition $|e\rangle_{c} \leftrightarrow|f\rangle_{c}$ is driven resonantly by an external classical field with Rabi frequency $\Omega_{C}(t)$. Then, we can write the interaction Hamiltonian of the whole system in the interaction picture as $(\hbar=1)$

$$
\begin{aligned}
& H_{I}(t)=H_{c}+H_{l}(t), \\
& H_{l}(t)=\Omega_{C}(t)|e\rangle_{c}\left\langle f\left|+\sum_{m=1}^{3} \Omega_{m}(t)\right| e\right\rangle_{m}\langle f|+H . c ., \\
& H_{c}=\sum_{m=1}^{3} \lambda_{m} a_{m}|e\rangle_{c}\left\langle g\left|+\sum_{m=1}^{3} v_{m} a_{m}\right| e\right\rangle_{m}\langle g|+H . c .,
\end{aligned}
$$

where $a_{m}$ is the photon annihilation operator of the $C P W R_{m}$. For simplicity, we assume $\lambda_{m}=v_{m}=\lambda, \Omega_{m}(t)=\Omega_{B}(t)$. Suppose that the system is initially in the state $\left|\Phi_{0}\right\rangle=$ $|f\rangle_{c}|g\rangle_{1}|g\rangle_{2}|g\rangle_{3}|0\rangle_{1}|0\rangle_{2}|0\rangle_{3}$, and the excited number operator of the system is defined as $N_{e}=$ $|e\rangle_{c}\langle e|+| f\rangle_{c}\langle f|+\sum_{m}\left(|e\rangle_{m}\langle e|+| f\rangle_{m}\langle f|+a_{m}^{\dagger} a_{m}\right)$. Due to $\left[N_{e}, H_{I}\right]=0$, and $\left\langle\Phi_{0}\left|N_{e}\right| \Phi_{0}\right\rangle=1$, the system will remain in the one-excited subspace spanned by

$$
\begin{aligned}
& \left|\psi_{1}\right\rangle=|f\rangle_{c}|g\rangle_{1}|g\rangle_{2}|g\rangle_{3}|0\rangle_{1}|0\rangle_{2}|0\rangle_{3},\left|\psi_{2}\right\rangle=|e\rangle_{c}|g\rangle_{1}|g\rangle_{2}|g\rangle_{3}|0\rangle_{1}|0\rangle_{2}|0\rangle_{3} \\
& \left|\psi_{3}\right\rangle=|g\rangle_{c}|g\rangle_{1}|g\rangle_{2}|g\rangle_{3}|1\rangle_{1}|0\rangle_{2}|0\rangle_{3},\left|\psi_{4}\right\rangle=|g\rangle_{c}|g\rangle_{1}|g\rangle_{2}|g\rangle_{3}|0\rangle_{1}|1\rangle_{2}|0\rangle_{3} \\
& \left|\psi_{5}\right\rangle=|g\rangle_{c}|g\rangle_{1}|g\rangle_{2}|g\rangle_{3}|0\rangle_{1}|0\rangle_{2}|1\rangle_{3},\left|\psi_{6}\right\rangle=|g\rangle_{c}|e\rangle_{1}|g\rangle_{2}|g\rangle_{3}|0\rangle_{1}|0\rangle_{2}|0\rangle_{3} \\
& \left|\psi_{7}\right\rangle=|g\rangle_{c}|g\rangle_{1}|e\rangle_{2}|g\rangle_{3}|0\rangle_{1}|0\rangle_{2}|0\rangle_{3},\left|\psi_{8}\right\rangle=|g\rangle_{c}|g\rangle_{1}|g\rangle_{2}|e\rangle_{3}|0\rangle_{1}|0\rangle_{2}|0\rangle_{3} \\
& \left|\psi_{9}\right\rangle=|g\rangle_{c}|f\rangle_{1}|g\rangle_{2}|g\rangle_{3}|0\rangle_{1}|0\rangle_{2}|0\rangle_{3},\left|\psi_{10}\right\rangle=|g\rangle_{c}|g\rangle_{1}|f\rangle_{2}|g\rangle_{3}|0\rangle_{1}|0\rangle_{2}|0\rangle_{3} \\
& \left|\psi_{11}\right\rangle=|g\rangle_{c}|g\rangle_{1}|g\rangle_{2}|f\rangle_{3}|0\rangle_{1}|0\rangle_{2}|0\rangle_{3}
\end{aligned}
$$


According to QZD, under the Zeno condition $\Omega_{B}(t) \ll \lambda$ and $\Omega_{C}(t) \ll \lambda$, the Hilbert subspace can be devided into seven invariant Zeno subspaces [68, 69]

$$
\begin{aligned}
& \Gamma_{P 1}=\left\{\left|\psi_{1}\right\rangle,\left|\Psi_{0}\right\rangle,\left|\psi_{9}\right\rangle,\left|\psi_{10}\right\rangle,\left|\psi_{11}\right\rangle\right\}, \\
& \Gamma_{P 2}=\left\{\left|\Psi_{1}\right\rangle\right\}, \Gamma_{P 3}=\left\{\left|\Psi_{2}\right\rangle\right\}, \\
& \Gamma_{P 4}=\left\{\left|\Psi_{3}\right\rangle\right\}, \Gamma_{P 5}=\left\{\left|\Psi_{4}\right\rangle\right\}, \Gamma_{P 6}=\left\{\left|\Psi_{5}\right\rangle\right\}, \Gamma_{P 7}=\left\{\left|\Psi_{6}\right\rangle\right\}
\end{aligned}
$$

where $\left|\Psi_{\iota}\right\rangle(\iota=0,1,2,3,4,5,6)$ is the eigenvector of the projections $P_{n^{\prime}}^{\alpha}$, which is defined as

$$
P_{n^{\prime}}^{\alpha}=|\alpha\rangle\langle\alpha|,\left(|\alpha\rangle \in \Gamma_{P n^{\prime}}\right)
$$

the corresponding eigenvectors

$$
\begin{aligned}
& \left|\Psi_{0}\right\rangle=\frac{1}{2}\left(-\left|\psi_{2}\right\rangle+\left|\psi_{6}\right\rangle+\left|\psi_{7}\right\rangle+\left|\psi_{8}\right\rangle\right), \\
& \left|\Psi_{1}\right\rangle=\frac{1}{2}\left(-\left|\psi_{3}\right\rangle+\left|\psi_{4}\right\rangle-\left|\psi_{6}\right\rangle+\left|\psi_{7}\right\rangle\right), \\
& \left|\Psi_{2}\right\rangle=\frac{1}{2}\left(-\left|\psi_{3}\right\rangle+\left|\psi_{5}\right\rangle-\left|\psi_{6}\right\rangle+\left|\psi_{8}\right\rangle\right), \\
& \left|\Psi_{3}\right\rangle=\frac{1}{2}\left(\left|\psi_{3}\right\rangle-\left|\psi_{4}\right\rangle-\left|\psi_{6}\right\rangle+\left|\psi_{7}\right\rangle\right), \\
& \left|\Psi_{4}\right\rangle=\frac{1}{2}\left(\left|\psi_{3}\right\rangle-\left|\psi_{5}\right\rangle-\left|\psi_{6}\right\rangle+\left|\psi_{8}\right\rangle\right), \\
& \left|\Psi_{5}\right\rangle=\frac{1}{2 \sqrt{6}}\left(3\left|\psi_{2}\right\rangle-2\left|\psi_{3}\right\rangle-2\left|\psi_{4}\right\rangle-2\left|\psi_{5}\right\rangle+\left|\psi_{6}\right\rangle+\left|\psi_{7}\right\rangle+\left|\psi_{8}\right\rangle\right), \\
& \left|\Psi_{6}\right\rangle=\frac{1}{2 \sqrt{6}}\left(3\left|\psi_{2}\right\rangle+2\left|\psi_{3}\right\rangle+2\left|\psi_{4}\right\rangle+2\left|\psi_{5}\right\rangle+\left|\psi_{6}\right\rangle+\left|\psi_{7}\right\rangle+\left|\psi_{8}\right\rangle\right),
\end{aligned}
$$

with corresponding eigenvalues $\eta_{0}=0, \eta_{1}=\lambda, \eta_{2}=\lambda, \eta_{3}=-\lambda, \eta_{4}=-\lambda, \eta_{5}=-2 \lambda$ and $\eta_{6}=2 \lambda$, respectively. So, the $H_{I}$ in Eq. (4) can be approximately expressed as [69]

$$
\begin{aligned}
H_{I} & \simeq \sum_{n^{\prime}, \alpha, \beta} \eta_{n^{\prime}} P_{n^{\prime}}^{\alpha}+P_{n^{\prime}}^{\alpha} H_{l} P_{n^{\prime}}^{\beta} \\
& =\lambda\left|\Psi_{1}\right\rangle\left\langle\Psi_{1}|+\lambda| \Psi_{2}\right\rangle\left\langle\Psi_{2}|-\lambda| \Psi_{3}\right\rangle\left\langle\Psi_{3}|-\lambda| \Psi_{4}\right\rangle\left\langle\Psi_{4}|-2 \lambda| \Psi_{5}\right\rangle\left\langle\Psi_{5}|+2 \lambda| \Psi_{6}\right\rangle\left\langle\Psi_{6}\right|
\end{aligned}
$$




$$
+\left(\Omega_{C}(t)\left|\psi_{2}\right\rangle\left\langle\psi_{1}\left|+\Omega_{B}(t)\right| \psi_{9}\right\rangle\left\langle\psi_{6}\left|+\Omega_{B}(t)\right| \psi_{10}\right\rangle\left\langle\psi_{7}\left|+\Omega_{B}(t)\right| \psi_{11}\right\rangle\left\langle\psi_{8}\right|+\text { H.c. }\right) .
$$

If the initial state is $\left|\Phi_{0}\right\rangle=|f\rangle_{c}|g\rangle_{1}|g\rangle_{2}|g\rangle_{3}|0\rangle_{1}|0\rangle_{2}|0\rangle_{3}$, then $H_{I}$ can reduce to

$$
\begin{aligned}
H_{e f f}(t) & =\left(-\frac{1}{2} \Omega_{C}(t)\left|\Psi_{0}\right\rangle\left\langle\psi_{1}\left|+\frac{\sqrt{3}}{2} \Omega_{B}(t)\right| \Psi_{0}\right\rangle\langle W|\right)+H . c . \\
& =\Omega_{I}(t)|W\rangle\left\langle\Psi_{0}\left|-\Omega_{I I}(t)\right| \psi_{1}\right\rangle\left\langle\Psi_{0}\right|+H . c .
\end{aligned}
$$

where $\Omega_{I}(t)=\sqrt{3} \Omega_{B}(t) / 2, \Omega_{I I}(t)=\Omega_{C}(t) / 2$, and $|W\rangle=\frac{1}{\sqrt{3}}\left(\left|\psi_{9}\right\rangle+\left|\psi_{10}\right\rangle+\left|\psi_{11}\right\rangle\right)$ is the $W$ state called the target state. Suppose that $\Omega_{I}(t)=\Omega(t) \cos \theta(t)$ and $\Omega_{I I}(t)=\Omega(t) \sin \theta(t)$, we can obtain the three instantaneous eigenstates of $H_{\text {eff }}(t)$ as follows:

$$
\begin{aligned}
& \left|\varphi_{0}(t)\right\rangle=\cos \theta\left|\psi_{1}\right\rangle+\sin \theta|W\rangle, \\
& \left|\varphi_{+}(t)\right\rangle=\frac{1}{\sqrt{2}}\left(\sin \theta\left|\psi_{1}\right\rangle-\left|\Psi_{0}\right\rangle-\cos \theta|W\rangle\right), \\
& \left|\varphi_{-}(t)\right\rangle=\frac{1}{\sqrt{2}}\left(\sin \theta\left|\psi_{1}\right\rangle+\left|\Psi_{0}\right\rangle-\cos \theta|W\rangle\right),
\end{aligned}
$$

with corresponding eigenvalues $\epsilon_{0}=0, \epsilon_{+}(t)=\Omega(t)$ and $\epsilon_{-}(t)=-\Omega(t)$, respectively. Choosing boundary condition $\theta(0)=0$ and $\theta(T)=\pi / 2$, generically, according to adiabatic state transfer, the initial state $\left|\psi_{1}\right\rangle$ can be transferred to the target state $|W\rangle$ by $\left|\varphi_{0}(t)\right\rangle$.

Through the unitary operator $U(t)=\sum_{r=0,+,-}\left|\varphi_{r}(t)\right\rangle\langle n|$, in the time-independent frame the $H_{\text {eff }}(t)$ is

$$
H_{a d}(t)=\Omega(t) M_{z}+\dot{\theta}(t) M_{y}
$$

in which $M_{z}=\left|\varphi_{+}\right\rangle\left\langle\varphi_{+}|-| \varphi_{-}\right\rangle\left\langle\varphi_{-}\left|, M_{y}=i\left(\left|\varphi_{+}\right\rangle+\left|\varphi_{-}\right\rangle\right)\left\langle\varphi_{0}\right| / \sqrt{2}+H . c .\right.\right.$, and $M_{x}=\left(\left|\varphi_{-}\right\rangle-\right.$ $\left.\left|\varphi_{+}\right\rangle\right)\left\langle\varphi_{0}\right| / \sqrt{2}+$ H.c.. As mentioned in Ref. [45], it is feasible to move the adiabatic frame to the frame defined by $V(t)$

$$
V(t)=\exp \left[i \eta(t) M_{z}\right] \exp \left[i \mu(t) M_{x}\right] \exp \left[i \xi(t) M_{z}\right]
$$

which is parametrized as a rotation of spin with Euler angles $\xi(t), \mu(t)$, and $\eta(t)$. Furthermore, the angle $\mu(t)$ should meet $\mu(0)=\mu(T)=0(2 \pi)$ to satisfy the condition 
$V(0)=V(T)=1$, and the other two angles can take any values. For making the form of correction Hamiltonian $H_{c o}(t)$ is similar to $H_{e f f}(t)$, the general form of $H_{c o}(t)$ is chosen

$$
H_{c o}(t)=U(t)\left(g_{x}(t) M_{x}+g_{z}(t) M_{z}\right) U^{\dagger}(t)
$$

in which $g_{x}(t)$ and $g_{z}(t)$ are two controlled parameters. So, the modified angle $\tilde{\theta}(t)$ and amplitude $\tilde{\Omega}(t)$ become

$$
\begin{aligned}
& \tilde{\theta}(t)=\theta(t)-\arctan \left(\frac{g_{x}(t)}{\Omega(t)+g_{z}(t)}\right) \\
& \tilde{\Omega}(t)=\sqrt{\left(\Omega(t)+g_{z}(t)\right)^{2}+g_{x}^{2}(t)} .
\end{aligned}
$$

Moreover, in order to cancel the unwanted transitions between dressed states, the control parameters should be selected as

$$
\begin{aligned}
& g_{x}(t)=\frac{\dot{\mu}}{\cos \xi}-\dot{\theta} \tan \xi \\
& g_{z}(t)=-\Omega+\dot{\xi}+\frac{\dot{\mu} \sin \xi-\dot{\theta}}{\tan \mu \cos \xi}
\end{aligned}
$$

in which, $g_{x}(t)$ and $g_{z}(t)$ are not depended on $\eta(t)$. In addition, the population of the intermediate state $\left|\Psi_{0}\right\rangle$ is expressed as

$$
\left|\left\langle\Phi(t) \mid \Psi_{0}(t)\right\rangle\right|=\sin ^{2} \mu(t) \cos ^{2} \xi(t)
$$

in which $|\Phi(t)\rangle$ is the state of the system. For keeping the discussion simple, we choose $\xi(t) \equiv 0$. The parameters are chosen as

$$
\begin{aligned}
& \mu(t)=\frac{A t^{2}}{2 T^{2}}\left[1-2\left(\frac{t}{T}\right)+\left(\frac{t}{T}\right)^{2}\right], \\
& \dot{\mu}(t)=\frac{A t}{T^{2}}\left[1-3\left(\frac{t}{T}\right)+2\left(\frac{t}{T}\right)^{2}\right], \\
& \theta(t)=\frac{\pi t^{4}}{2 T^{4}}\left[35-84\left(\frac{t}{T}\right)+70\left(\frac{t}{T}\right)^{2}-20\left(\frac{t}{T}\right)^{3}\right], \\
& \dot{\theta}(t)=\frac{70 \pi t^{3}}{T^{4}}\left[1-2\left(\frac{t}{T}\right)+\left(\frac{t}{T}\right)^{2}\right]\left(1-\frac{t}{T}\right),
\end{aligned}
$$

where $A$ is a time-independent parameter controlling the maximal value of $\mu(t)$, the boundary condition $\mu(0)=\mu(T)=0(2 \pi), \theta(0)=0$ and $\theta(T)=\pi / 2$, is well satisfied for the 
choosing parameters. The population of intermediate state $\left|\Psi_{0}\right\rangle$ and the interaction time $T$ are affected by the parameter $A$. So, what we need is to choose a suitable $A$ to restrict the interaction time and population of intermediate state in an expected range. We find that $A=14$ can meet the requirement and we can achieve $\left|\left\langle\Phi(t) \mid \Psi_{0}(t)\right\rangle\right|=\sin ^{2} \mu(t) \leq 0.18$, $\tilde{\Omega}(t) \times T \leq 7$. Furthermore, according to the Eq. (18), the pulses $\tilde{\Omega}_{I}(t)=\tilde{\Omega}(t) \cos \tilde{\theta}(t)$ and $\tilde{\Omega}_{I I}(t)=\tilde{\Omega}(t) \sin \tilde{\theta}(t)$ are so complicated. For experimental feasibility, we substitute pulses $\tilde{\Omega}_{I}(t)$ and $\tilde{\Omega}_{I I}(t)$ by $\bar{\Omega}_{I}(t)$ and $\bar{\Omega}_{I I}(t)$, respectively,

$$
\begin{aligned}
& \bar{\Omega}_{I}(t)=\chi_{a_{1}} e^{-\left[\left(t-\tau_{a_{1}}\right) / \sigma_{a_{1}}\right]^{2}}+\chi_{a_{2}} e^{-\left[\left(t-\tau_{a_{2}}\right) / \sigma_{a_{2}}\right]^{2}}, \\
& \bar{\Omega}_{I I}(t)=\chi_{b_{1}} e^{-\left[\left(t-\tau_{b_{1}}\right) / \sigma_{b_{1}}\right]^{2}}+\chi_{b_{2}} e^{-\left[\left(t-\tau_{b_{2}}\right) / \sigma_{b_{2}}\right]^{2}},
\end{aligned}
$$

in which,

$$
\begin{gathered}
\chi_{a_{1}}=4.28 / T, \chi_{a_{2}}=3.222 / T, \chi_{b_{1}}=4.28 / T, \chi_{b_{2}}=3.222 / T \\
\tau_{a_{1}}=0.2914 T, \tau_{a_{2}}=0.5285 T, \tau_{b_{1}}=0.7085 T, \tau_{b_{2}}=0.4715 T \\
\sigma_{a_{1}}=0.227 T, \sigma_{a_{2}}=0.3181 T, \sigma_{b_{1}}=0.227 T, \sigma_{b_{2}}=0.3181 T
\end{gathered}
$$

$\bar{\Omega}_{I}(t)$ and $\bar{\Omega}_{I I}(t)$ are the linear superposition of Gaussian pulses. We plot the $\tilde{\Omega}_{I}(t)\left(\tilde{\Omega}_{I I}(t)\right)$ and $\bar{\Omega}_{I}(t)\left(\bar{\Omega}_{I I}(t)\right)$ versus $t / T$ in Fig. 2. Seen from Fig. $2, \bar{\Omega}_{I}(t)\left(\bar{\Omega}_{I I}(t)\right)$ and $\tilde{\Omega}_{I}(t)\left(\tilde{\Omega}_{I I}(t)\right)$ match well. In the following section, we will demonstrate the substitutions are valid and the system can be driven from its initial state $\left|\Phi_{0}\right\rangle=\left|\psi_{1}\right\rangle$ to the target state $|\Phi(T)\rangle=|W\rangle$ by the Rabi frequencies $\bar{\Omega}_{I}(t)=\sqrt{3} \bar{\Omega}_{B}(t) / 2$ and $\bar{\Omega}_{I I}(t)=\bar{\Omega}_{C}(t) / 2$ with high fidelity.

\section{NUMERICAL SIMULATIONS AND FEASIBILITY ANALYSIS}

In this section, we will investigate the feasibility and robustness of the scheme with numerical simulations. We define the fidelity of the target state $|W\rangle$ as $F(t)=|\langle W|\rho(t)| W\rangle|$, in which $\rho(t)$ is the density operator of the system. Then, we plot the final fidelity $F(T)$ versus $\lambda$ in Fig. 3. The figure shows that when $\lambda \geq 10 / T, F(t)$ is close to 1 . In addition, the effective Hamiltonian $H_{\text {eff }}(t)$ is obtained under the Zeno condition $\bar{\Omega}_{B}(t) \ll \lambda$ and $\bar{\Omega}_{C}(t) \ll \lambda$ in Sec. III. Therefore, it is necessary to adopt a suitable $\lambda$. From Fig. 2, 
the max amplitude of the pulse is $\bar{\Omega}_{0}=\max _{0 \leq t \leq T}\left\{\bar{\Omega}_{B}(t), \bar{\Omega}_{C}(t)\right\} \approx 14 / T$, and $\bar{\Omega}_{0} \ll \lambda$ can substitute $\bar{\Omega}_{B}(t) \ll \lambda$ and $\bar{\Omega}_{C}(t) \ll \lambda$. As it's known, if $\lambda$ is not enough large, the system will suffer more decoherence because of the high populations of intermediate states. Also, the larger the $\lambda$, the better the Zeno condition is satisfied. But, the coupling constant $\lambda$ has an upper limit in real experiments that would limit the speed of the system's evolution. So, considering the high speed and robustness of the scheme, we choose $\lambda=20 / T$ that a little larger than $\bar{\Omega}_{0}$. With the suitable $\lambda$, we investigate the fidelity $F(t)$ and the population $P_{k}=\left\langle\psi_{k}|\rho(t)| \psi_{k}\right\rangle(k=1,2,3, \ldots, 11)$ of state $\left|\psi_{k}\right\rangle$ during the evolution in Fig. 4. Fig. 4 (a) shows the final fidelity can reach 1 . As shown in Fig. 4 (b), the green solid line including populations $P_{3}, P_{4}$ and $P_{5}$ are nearly close to 0 during the evolution, which shows $\left|\psi_{3}\right\rangle,\left|\psi_{4}\right\rangle$, and $\left|\psi_{5}\right\rangle$ are not the component of the $\left|\Phi_{0}\right\rangle$. So, the $H_{e f f}(t)$ of the system is valid. The Fig. 4 (b) also shows the target state $|W\rangle$ can be obtained at $t=T$ and the intermediate states are slightly populated based on the present scheme.

We find the form of $H_{e f f}(t)$ is similar to stimulated Raman adiabatic passage (STIRAP) techniques. Therefore, we make a comparison between the scheme and that with STIRAP. The Rabi frequencies of pulses via STIRAP are chosen as

$$
\Omega_{B}^{\prime}(t)=\Omega_{0}^{\prime} e^{-\left[(t-t 0-T / 2) / t_{c}\right]^{2}}, \Omega_{C}^{\prime}(t)=\Omega_{0}^{\prime} e^{-\left[(t+t 0-T / 2) / t_{c}\right]^{2}}
$$

where, $\Omega_{0}^{\prime}$ is the pulse amplitude for STIRAP, $t_{0}=0.15 T$ and $t_{c}=0.2 T$ are the two related parameters. We plot the fidelity of the target state $|W\rangle$ with different methods versus $\mathrm{t} / \mathrm{T}$ in Fig. 5. The pink solid line shows the final fidelity of the present scheme can reach 1 with $\Omega_{0}^{\prime}=14 / T$ and $\lambda=20 / T$, whereas at the same conditions, the final fidelity of the scheme with STIRAP is only about 0.2 (see the green solid line in Fig. 5) due to the greatly violation of the adiabatic condition. As shown in Fig. 5, for the STIRAP method with the increasing of the $\Omega_{0}^{\prime}$ and $\lambda$, the fidelity also increases. When $\Omega_{0}^{\prime}=80 / T$ and $\lambda=240 / T$ (see the blue solid line in Fig. 5) the fidelity can approach 1. However, in this case, the laser amplitude $\Omega_{0}^{\prime}=80 / T$ is much larger than the one $\left(\bar{\Omega}_{0} \approx 14 / T\right)$ of the scheme. Therefore, compared with the STIRAP method, the scheme requires less physical resources and is fast a lot to achieve the target state.

In the above discussion, we haven't considered the dissipation caused by decoherence mechanisms. However, the system will inevitably interact with the environment, which affects the usability of this scheme. Here, the influence of different kinds of dissipative 
factors should be investigated, the decay of the cavity mode in each $C P W R$, the energy relaxation and the dephasing of every $S Q$. Considering these influence, the evolution of the system can be modeled by a master equation in Lindblad form

$$
\dot{\rho}=i\left[\rho, H_{I}\right]+\sum_{l}\left[L_{l} \rho L_{l}^{\dagger}-\frac{1}{2}\left(L_{l}^{\dagger} L_{l} \rho+\rho L_{l}^{\dagger} L_{l}\right)\right]
$$

in which, $L_{l}$ is the Lindblad operator. There exists nineteen Lindblad operators

$$
\begin{aligned}
& L_{1}=\sqrt{\gamma_{1 f}}|f\rangle_{1}\left\langle e\left|, \quad L_{2}=\sqrt{\gamma_{2 f}}\right| f\right\rangle_{2}\left\langle e\left|, \quad L_{3}=\sqrt{\gamma_{3 f}}\right| f\right\rangle_{3}\left\langle e\left|, \quad L_{4}=\sqrt{\gamma_{c f}}\right| f\right\rangle_{c}\langle e|, \\
& L_{5}=\sqrt{\gamma_{1 g}}|g\rangle_{1}\left\langle e\left|, \quad L_{6}=\sqrt{\gamma_{2 g}}\right| g\right\rangle_{2}\left\langle e\left|, \quad L_{7}=\sqrt{\gamma_{3 g}}\right| g\right\rangle_{3}\left\langle e\left|, \quad L_{8}=\sqrt{\gamma_{c g}}\right| g\right\rangle_{c}\langle e|, \\
& L_{9}=\sqrt{\kappa_{1}} a_{1}, \quad L_{10}=\sqrt{\kappa_{2}} a_{2}, \quad L_{11}=\sqrt{\kappa_{3}} a_{3}, \\
& L_{12}=\sqrt{\gamma_{\phi 1 f} / 2}\left(|e\rangle_{1}\langle e|-| f\rangle_{1}\langle f|\right), \quad L_{13}=\sqrt{\gamma_{\phi 2 f} / 2}\left(|e\rangle_{2}\langle e|-| f\rangle_{2}\langle f|\right), \\
& L_{14}=\sqrt{\gamma_{\phi 3 f} / 2}\left(|e\rangle_{3}\langle e|-| f\rangle_{3}\langle f|\right), \quad L_{15}=\sqrt{\gamma_{\phi c f} / 2}\left(|e\rangle_{c}\langle e|-| f\rangle_{c}\langle f|\right), \\
& L_{16}=\sqrt{\gamma_{\phi 1 g} / 2}\left(|e\rangle_{1}\langle e|-| g\rangle_{1}\langle g|\right), \quad L_{17}=\sqrt{\gamma_{\phi 2 g} / 2}\left(|e\rangle_{2}\langle e|-| g\rangle_{2}\langle g|\right), \\
& L_{18}=\sqrt{\gamma_{\phi 3 g} / 2}\left(|e\rangle_{3}\langle e|-| g\rangle_{3}\langle g|\right), \quad L_{19}=\sqrt{\gamma_{\phi c g} / 2}\left(|e\rangle_{c}\langle e|-| g\rangle_{c}\langle g|\right),
\end{aligned}
$$

where, $\gamma_{m s}(m=1,2,3, s=f, g)$ is the energy relaxation rate and $\gamma_{\phi m s}$ is the dephasing rate of the $m$-th SQ for decay path $|e\rangle_{m} \rightarrow|s\rangle_{m}$, respectively. And $\gamma_{c s}(s=f, g)$ is the energy relaxation rate and $\gamma_{\phi c s}$ is the dephasing rate of the SCC qubit for decay path $|e\rangle_{c} \rightarrow|s\rangle_{c} . \kappa_{m}$ is the decay rate of the $m$-th cavity mode in $C P W R_{m}$. For simplicity, assuming $\gamma_{m s}=\gamma_{c s}=\gamma, \gamma_{\phi m s}=\gamma_{\phi c s}=\gamma_{\phi}$ and $\kappa_{m}=\kappa$, we plot the final fidelity $F(T)$ versus $\gamma_{\phi} / \lambda, \gamma / \lambda$ and $\kappa / \lambda$ in Fig. 6. From Fig. 6 (b) and Fig. 6 (c), we can find that the present scheme is robust against the decay of the cavity modes in $C P W R_{m}$. Moreover, from Fig. 6 (a) and Fig. 6 (c), we also can see that the scheme is robust to energy relaxation rate. However, from Fig. 6 we can see that the fidelity is mainly affected dephasing rate. To intuitively investigate the performance of the depasing of $S Q_{s}$ and SCC, some values of $F(T)$ versus $\kappa / \lambda, \gamma / \lambda$ and $\gamma_{\phi} / \lambda$ are listed in Table I. From the Table I, when the $\kappa / \lambda=0.001$, $\gamma_{\phi} / \lambda=0.001$ and $\gamma / \lambda=0.001$ change to $\kappa / \lambda=0.1, \gamma_{\phi} / \lambda=0.1$ and $\gamma / \lambda=0.1$, the $F(T)$ 
changes by only 0.0355 from 0.9947 to 0.9592 . We also list other situations in the Table I. All these results show the present scheme is robust to the dissipation.

Finally, because most of the parameters are hard to faultlessly achieved in the experiments, it is necessary to investigate the variations of the parameters caused by the experimental imperfection. Therefore, we investigate the influence of the variations in the interaction time $T$, the coupling constant $\lambda$ and the pulse amplitude $\bar{\Omega}_{0}$. The $T^{\prime}=T+\delta T$ is defined as the erroneous total interaction time when the original time exists a variation $\delta T$. We depict fidelity $F\left(t^{\prime}\right)$ versus $\delta \lambda / \lambda, \delta \bar{\Omega}_{0} / \bar{\Omega}_{0}$ and $\delta T / T$ in Fig. 7. From Fig. 7 (a) and Fig. 7 (c), one can see that fidelity $F\left(T^{\prime}\right)$ is nearly not affected by the $\delta \bar{\Omega}_{0} / \bar{\Omega}_{0}$ and $\delta \lambda / \lambda$ but is mainly influenced by $\delta T / T$. To exhibit clearly the influence of $\delta T / T$, in Table II we list some samples of the $F\left(T^{\prime}\right)$ versus $\delta \lambda / \lambda, \delta \bar{\Omega}_{0} / \bar{\Omega}_{0}$ and $\delta T / T$. From the Table, for $T^{\prime}=0.9 T$ or $T^{\prime}=1.1 T$ (i.e., $10 \%$ interaction time error), the $F\left(T^{\prime}\right)$ drops down to $\leq 0.06$. So, the $F\left(T^{\prime}\right)$ is insensitive to the interaction time. Therefore, the present scheme is robust to the variations $\delta \lambda, \delta \bar{\Omega}_{0}$, and $\delta T$.

\section{CONCLUSION}

We have presented an efficient scheme for fast generating $W$ state of the three superconducting qubits by using the quantum Zeno dynamins and the dressed states. Choosing a set of dressed states suitable, two practical pulses in experiment can be designed for accelerating the evolution of the system. Numerical simulations show the scheme is feasible in practice and the $W$ state can be rapidly achieved, the interaction time of scheme required for generating the target state by using dressed states is shorter than that via adiabatic passage. Moreover, we have discussed the influence of deviations of various parameters and the various decoherence processes on the fidelity of the target state, which shows the present scheme is robust to the influence. The scheme may be experimentally implementable because of the remarkable progress in circuit quantum electrodynamics.

\section{Acknowledgment}

This work was supported by the National Natural Science Foundation of China under Grants No. 11575045, No. 11374054 and No. 11674060, and the Major State Basic Research 
Development Program of China under Grant No. 2012CB921601.

[1] A. Rauschenbeutel, G. Nogues, S. Osnaghi, P. Bertet, M. Brune, J. M. Raimond, and S. Haroche, Science 288, 2024 (2000).

[2] J. W. Pan, M. Daniell, S. Gasparoni, G. Weihs, and A. Zeilinger, Phys. Rev. Lett. 86, 4435 (2001).

[3] C. F. Roos, M. Riebe, H. Häffner, W. Hänsel, J. Benhelm, G. P. T. Lancaster, C. Becher, F. Schmidt-Kaler, and R. Blatt, Science 304, 1097522 (2004).

[4] Q. C. Wu and X. Ji, Quantum Inf. Process. 12, 3167 (2013).

[5] A. Wallraff, D. I. Schuster, A. Blais, L. Frunzio, R. S. Huang, J. Majer, S. Kumar, S. M. Girvin, and R. J. Schoelkopf, Nature 431, 162 (2004).

[6] A. Blais, J. Gambetta, A. Wallraff, D. I. Schuster, S. M. Girvin, M. H. Devoret, and R. J. Schoelkopf, Phys. Rev. A 75, 032329 (2007).

[7] L. Frunzio, A. Wallraff, D. Schuster, J. Majer, and R. J. Schoelkopf, IEEE Trans. Appl. Supercond. 15, $860(2005)$.

[8] K. D. Petersson, L. W. McFaul, M. D. Schroer, M. Jung, J. M. Taylor, A. A. Houck, and J. R. Petta, Nature 490, 380 (2012).

[9] T. D. Ladd, P. van Loock, K. Nemoto, W. J. Munro, and Y. Yamamoto, New J. Phys. 8, 184 (2006).

[10] C. P. Yang, S. I. Chu and S. Han, Phys. Rev. A 67, 042311 (2003).

[11] C. P. Yang, S. I. Chu and S. Han, Phys. Rev. Lett. 92, 117902 (2004).

[12] C. P. Yang, and S. Han, Phys. Rev. A 74, 044302 (2006).

[13] C. P. Yang, Q. P. Su and S. Han, Phys. Rev. A 86, 022329 (2012).

[14] J. Koch, T. M. Yu, J. Gambetta, A. A. Houck, D. I. Schuster, J. Majer, A. Blais, M. H. Devoret, S. M. Girvin, and R. J. Schoelkopf, Phys. Rev. A 76, 042319 (2007).

[15] A. Blais, R. S. Huang, A. Wallraff, S. M. Girvin, and R. J. Schoelkopf, Phys. Rev. A 69, $062320(2004)$.

[16] R. J. Schoelkopf and S. M. Girvin, Nature 451, 664 (2008).

[17] S. M. Girvin, M. H. Devoret, and R. J. Schoelkopf, Phys. Scr. T137, 014012 (2009).

[18] Y. Makhlin, G. Schön, and A. Shnirman, Rev. Mod. Phys. 73, 357 (2001). 
[19] Y. X. Liu, L. F. Wei, and F. Nori, Europhys. Lett. 67, 941 (2004).

[20] K. H. Song, Z. W. Zhou, and G. C. Guo, Phys. Rev. A 71, 052310 (2005).

[21] Z. J. Deng, K. L. Gao, and M. Feng, Phys. Rev. A 74, 064303 (2006).

[22] Y. D. Wang, S. Chesi, D. Loss, and C. Bruder, Phys. Rev. B 81, 104524 (2010).

[23] K. H. Song, S. H. Xiang, Q. Liu and D. H. Lu, Phys. Rev. A 75, 032347 (2007).

[24] W. Feng, P. Wang, X. Ding, L. Xu, and X. Q. Li, Phys. Rev. A 83, 042313 (2011).

[25] C. P. Yang, Phys. Rev. A 83, 062302 (2011).

[26] F. W. Strauch, K. Jacobs, and R. W. Simmonds, Phys. Rev. Lett. 105, 050501 (2010).

[27] C. P. Yang, Q. P. Su, S. B. Zheng, and S. Y. Han, Phys. Rev. A 87, 022320 (2013).

[28] D. M. Greenberger, M. A. Horne, and A. Zeilinger, in Bell's Theorem, Quantum Theory, and Conceptions of the Universe, edited by M. Kafatos (Kluwer, Dordrecht, 1989).

[29] W. Dür, G. Vidal, and J. I. Cirac, Phys. Rev. A 62, 062314 (2000).

[30] X. Wei and M. F. Chen, Int. J. Theor. Phys. 54, 812 (2015).

[31] X. Wei and M. F. Chen, Quantum Inf. Process. 14, 2419 (2015).

[32] Y. H. Chen, B. H. Huang, J. Song, and Y. Xia, Opt. Commun. 380, 140 (2016).

[33] Y. H. Chen, Y. Xia, Q. Q. Chen, and J. Song, Laser Phys. Lett. 11, 115201 (2014).

[34] M. Demirplak and S. A. Rice, J. Phys. Chem. A 107, 9937 (2003).

[35] M. Demirplak and S. A. Rice, J. Chem. Phys. 129, 154111 (2008).

[36] M. V. Berry, J. Phys. A 42, 365303 (2009).

[37] J. G. Muga, X. Chen, A. Ruschhaupt, and D. Guéry-Odelin, J. Phys. B 42, 241001 (2009).

[38] X. Chen, A. Ruschhaupt, S. Schmidt, A. del Campo, D. Guéry-Odelin, and J. G. Muga, Phys. Rev. Lett. 104, 063002 (2010).

[39] X. Chen, I. Lizuain, A. Ruschhaupt, D. Guéry-Odelin, and J. G. Muga, Phys. Rev. Lett. 105, $123003(2010)$.

[40] X. Chen, E. Torrontegui, and J. G. Muga, Phys. Rev. A 83, 062116 (2011).

[41] A. del Campo and M. G. Boshier, Sci. Rep. 2, 648 (2012).

[42] X. Chen and J. G. Muga, Phys. Rev. A 86, 033405 (2012).

[43] E. Torrontegui, S. Ibáñez, S. Martínez-Garaot, M. Modugno, A. del Campo, D. Gué-Odelin, A. Ruschhaupt, X. Chen, and J. G. Muga, Adv. Atom. Mol. Opt. Phys. 62, 117 (2013).

[44] A. del Campo, Phys. Rev. Lett. 111, 100502 (2013).

[45] A. Baksic, H. Ribeiro, and A. A. Clerk, Phys. Rev. Lett. 116, 230503 (2016). 
[46] S. Ibáñez, X. Chen, and J. G. Muga, Phys. Rev. A 87, 043402 (2013).

[47] S. Ibáñez, X. Chen, E. Torrontegui, J. G. Muga, and A. Ruschhaupt, Phys. Rev. Lett. 109, 100403 (2012).

[48] B. T. Torosov, G. D. Valle, and S. Longhi, Phys. Rev. A 87, 052502 (2013).

[49] S. Martínez-Garaot, E. Torrontegui, X. Chen, and J. G. Muga, Phys. Rev. A 89, 053408 (2014).

[50] T. Opatrný and K. Mømer, New J. Phys. 16, 015025 (2014).

[51] H. Saberi, T. Opatrný, K. Mømer, and A. del Campo, Phys. Rev. A 90, 060301 (2014).

[52] E. Torrontegui, S. Martínez-Garaot, and J. G. Muga, Phys. Rev. A 89, 043408 (2014).

[53] B. T. Torosov, G. D. Valle, and S. Longhi, Phys. Rev. A 89, 063412 (2014).

[54] Y. H. Chen, Y. Xia, Q. C. Wu, B. H. Huang, and J. Song, Phys. Rev. A 93, 052109 (2016).

[55] X. K. Song, Q. Ai, J. Qiu, and F. G. Deng, Phys. Rev. A 93, 052324 (2016).

[56] X. K. Song, H. Zhang, Q. Ai, J. Qiu, and F. G. Deng, New J. Phys. 18, 023001 (2016).

[57] M. M. Rams, M. Mohseni, and A. del Campo, New J. Phys. 18, 123034 (2016).

[58] S. An, D. Lv, A. del Campo, and K. Kim, Nat. Commun. 7, 12999 (2016).

[59] A. del Campo, Phys. Rev. A 84, 031606 (2011).

[60] A. del Campo, M. M. Rams, and W. H. Zurek, Phys. Rev. Lett. 109, 115703 (2012).

[61] S. Deffner, C. Jarzynski, and A. del Campo, Phys. Rev. X 4, 021013 (2014).

[62] A. del Campo, Phys. Rev. Lett. 111, 100502 (2013).

[63] A. del Campo, Eur. Phys. Lett. 96, 60005 (2011).

[64] S. Ibáñez, S. Martínez-Garaot, X. Chen, E. Torrontegui and X. Chen, Phys. Rev. A 84, 023415 (2011).

[65] S. Ibáñez, Y. C. Li and X. Chen and J. G. Muga, Phys. Rev. A 92, 062136 (2015).

[66] A. del Campo, New J. Phys. 18, 015014 (2016).

[67] Y. Ban and X. Chen, Sci. Rep. 6, 6258 (2014).

[68] P. Facchi and S. Pascazio, Phys. Rev. Lett. 89, 080401 (2002).

[69] P. Facchi, G. Marmo, and S. Pascazio, J. Phys.: Conf. Ser. 196, 012017 (2009). 
Table I. Samples of the final fidelity $F(T)$ with corresponding $\kappa / \lambda, \gamma / \lambda$ and $\gamma_{\phi} / \lambda$.

\begin{tabular}{cccc}
\hline \hline$\kappa / \lambda\left(\times 10^{-2}\right)$ & $\gamma_{\phi} / \lambda\left(\times 10^{-3}\right)$ & $\gamma / \lambda\left(\times 10^{-2}\right)$ & $F$ \\
\hline 1 & 1 & 1 & 0.9592 \\
1 & 1 & 0.8 & 0.9644 \\
1 & 0.8 & 1 & 0.9613 \\
0.8 & 1 & 1 & 0.9596 \\
0.8 & 0.8 & 0.8 & 0.9669 \\
0.8 & 0.8 & 0.5 & 0.9748 \\
0.8 & 0.5 & 0.8 & 0.9701 \\
0.5 & 0.8 & 0.8 & 0.9676 \\
0.5 & 0.5 & 0.5 & 0.9787 \\
0.5 & 0.5 & 0.3 & 0.9840 \\
0.5 & 0.3 & 0.5 & 0.9809 \\
0.3 & 0.5 & 0.5 & 0.9791 \\
0.3 & 0.3 & 0.3 & 0.9867 \\
0.3 & 0.3 & 0.1 & 0.9921 \\
0.3 & 0.1 & 0.3 & 0.9889 \\
0.1 & 0.3 & 0.3 & 0.9871 \\
0.1 & 0.1 & 0.1 & 0.9947 \\
\hline \hline
\end{tabular}


Table II. Samples of the fidelity $F\left(T^{\prime}\right)$ with $\delta \bar{\Omega}_{0} / \bar{\Omega}_{0}, \delta \lambda / \lambda$ and $\delta T / T$.

\begin{tabular}{cccc}
\hline \hline$\delta \bar{\Omega}_{0} / \bar{\Omega}_{0}$ & $\delta \lambda / \lambda$ & $\delta T / T$ & $F\left(T^{\prime}\right)$ \\
\hline 0 & $-10 \%$ & 0 & 0.9985 \\
0 & $10 \%$ & 0 & 0.9993 \\
0 & 0 & $-5 \%$ & 0.9875 \\
0 & 0 & $5 \%$ & 0.9918 \\
0 & 0 & $10 \%$ & 0.9685 \\
$10 \%$ & $10 \%$ & $10 \%$ & 0.9685 \\
$-10 \%$ & $-10 \%$ & $-10 \%$ & 0.9454 \\
$-10 \%$ & $10 \%$ & $10 \%$ & 0.9685 \\
$10 \%$ & $-10 \%$ & $10 \%$ & 0.9683 \\
$10 \%$ & $10 \%$ & $-10 \%$ & 0.9478 \\
$-10 \%$ & $-10 \%$ & $10 \%$ & 0.9683 \\
$-10 \%$ & $10 \%$ & $-10 \%$ & 0.9478 \\
$10 \%$ & $-10 \%$ & $-10 \%$ & 0.9454 \\
\hline \hline
\end{tabular}



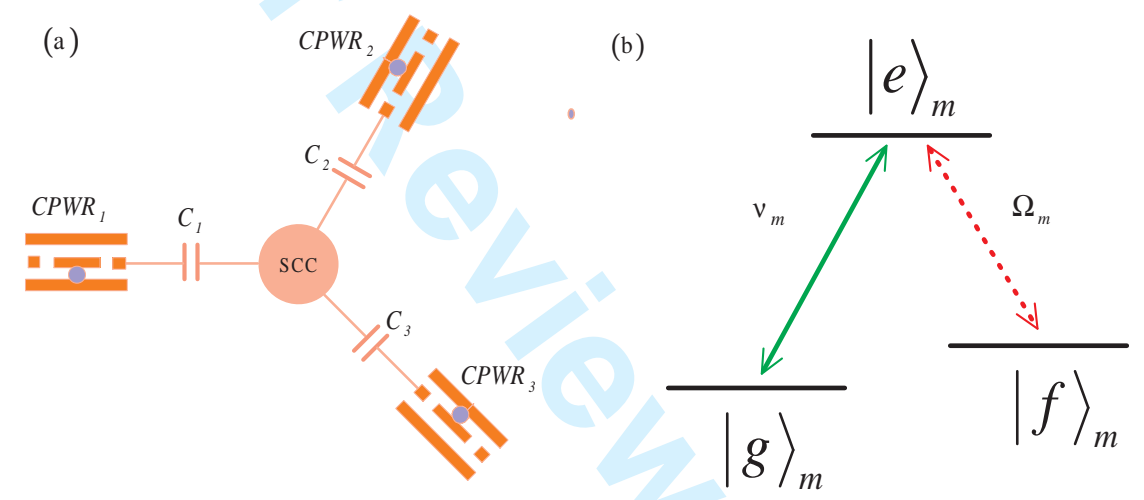

FIG. 1: (a)The schematic diagram of the $C P W R_{m}(m=1,2,3)$ and a single SCC qubit (a circle places in the center of the devices). (b)The energy-level structure of the $S Q_{m}$ qubit and relevant transitions. 

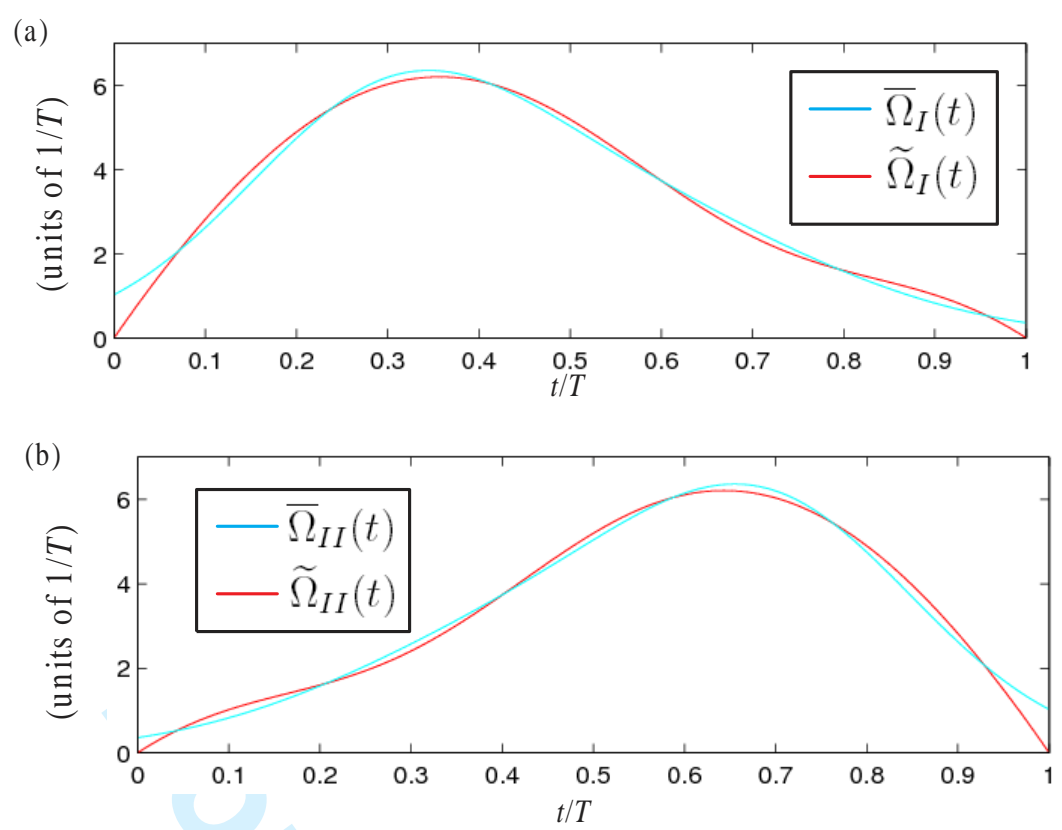

FIG. 2: (a) Comparison between $\widetilde{\Omega}_{I}(t)$ and $\bar{\Omega}_{I}(t)$ (versus $t / T$ ). (b) Comparison between $\widetilde{\Omega}_{I I}(t)$ and $\bar{\Omega}_{I I}(t)(\operatorname{versus} t / T)$.

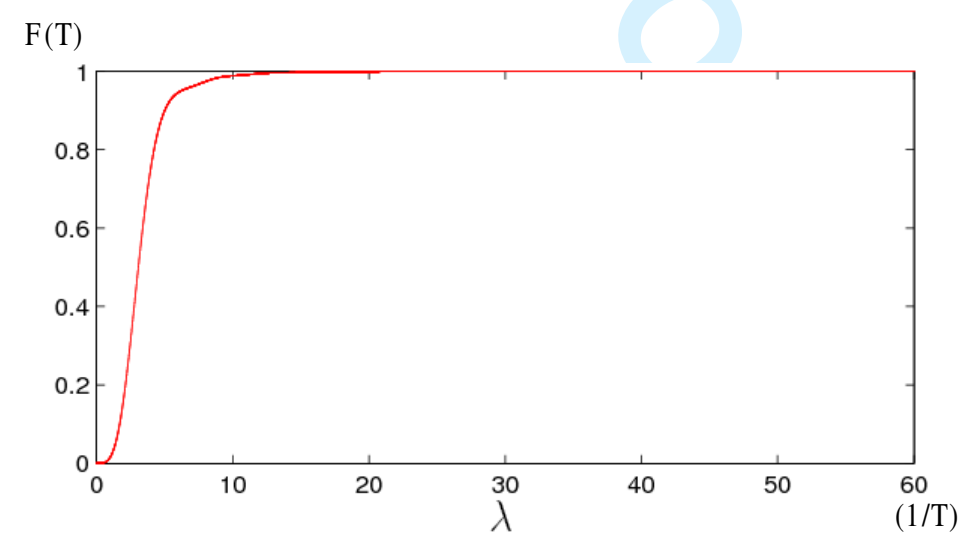

FIG. 3: The final fidelity $F(T)$ versus $\lambda$. 
(a)

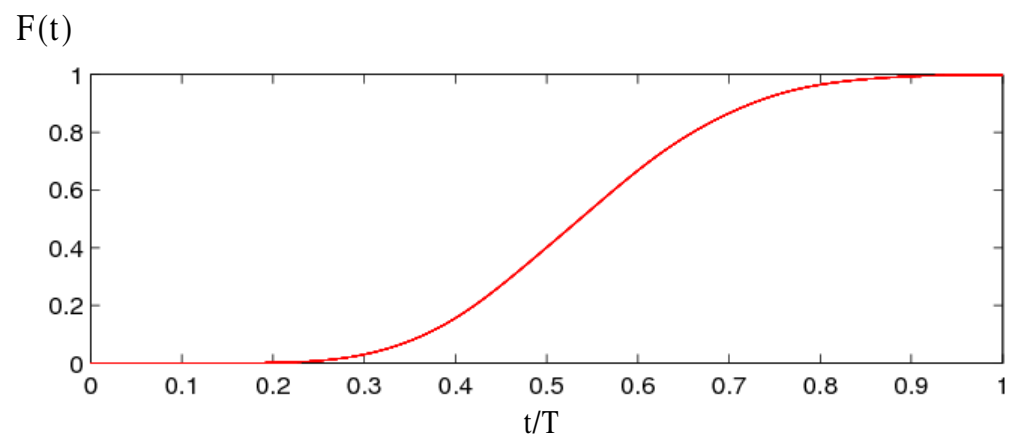

(b)

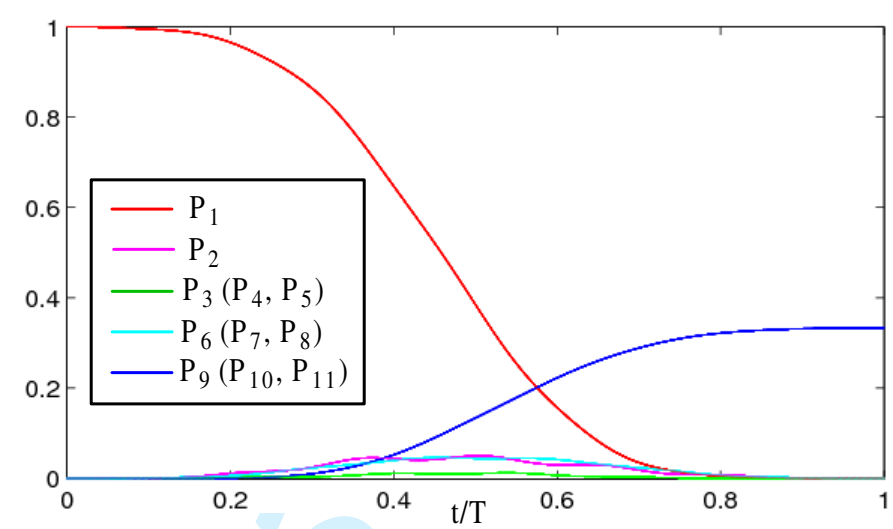

FIG. 4: (a) The fidelity $F(t)$ versus $t / T$. (b) The population $P_{m}$ of $\left|\psi_{m}\right\rangle$ versus $t / T$.

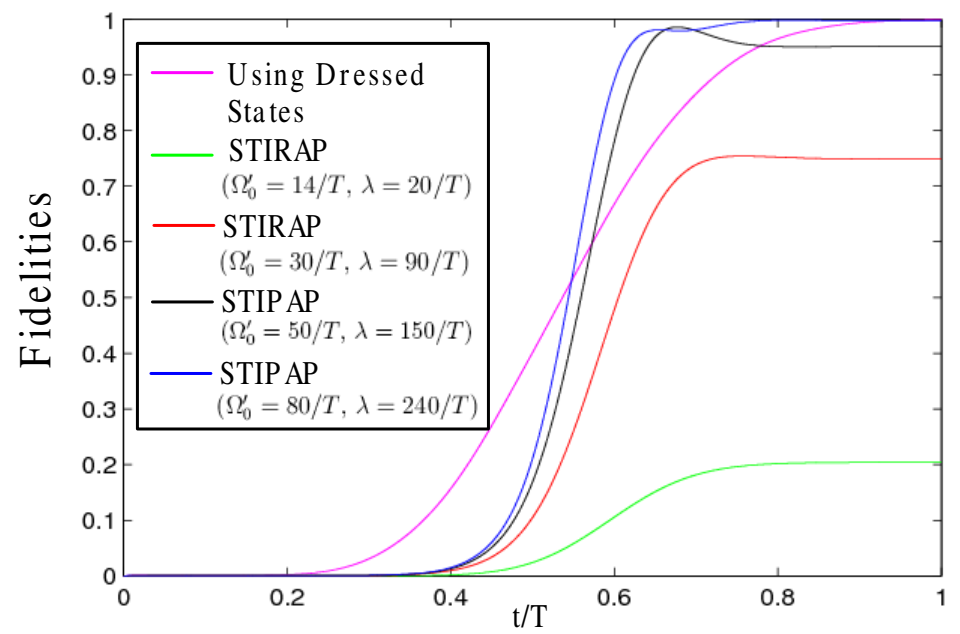

FIG. 5: The fidelities of the target state $|W\rangle$ versus $t / T$ with different methods. 

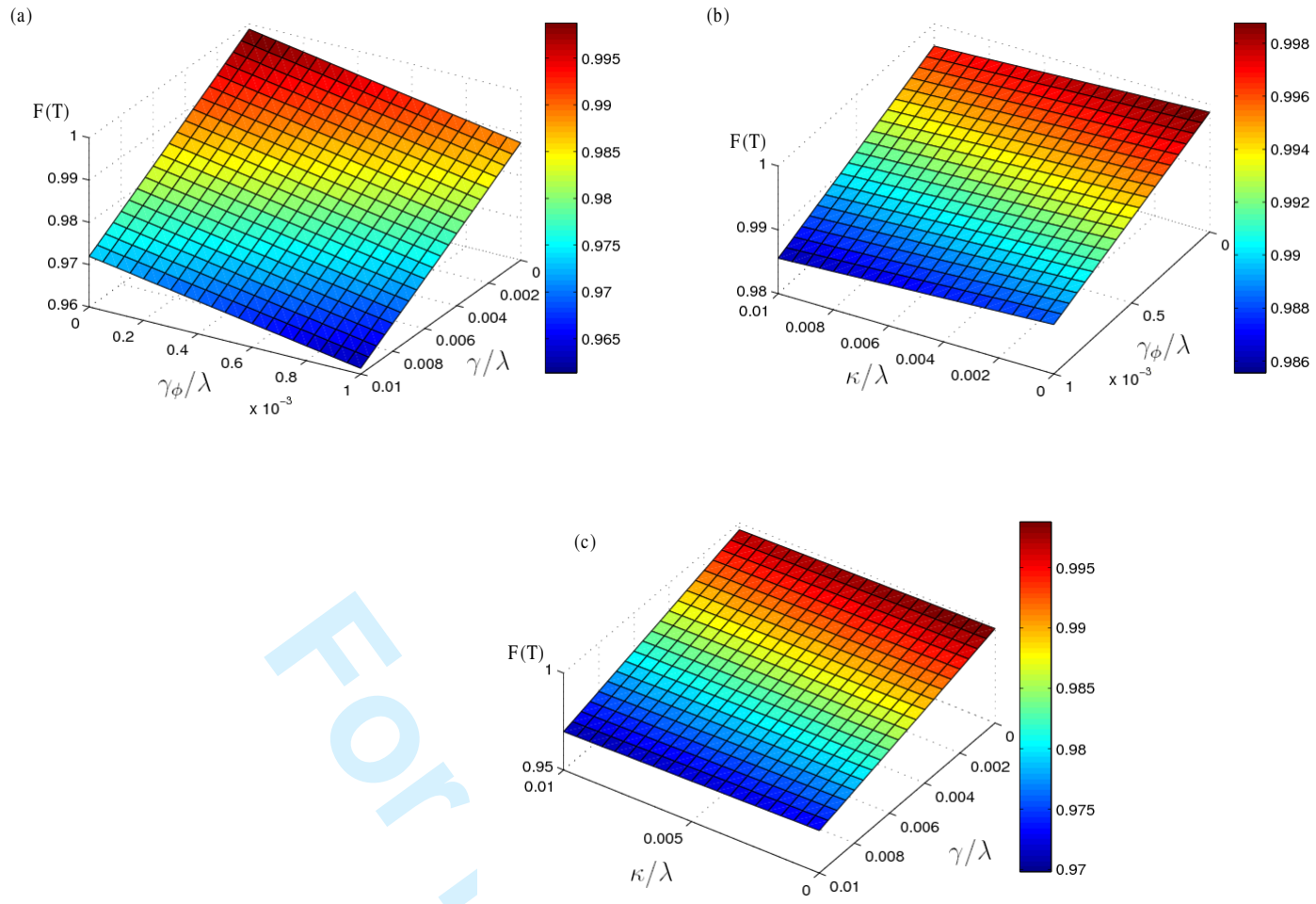

FIG. 6: (a) The final fidelity $F(T)$ versus $\gamma / \lambda$ and $\gamma_{\phi} / \lambda$. (b) The final fidelity $F(T)$ versus $\kappa / \lambda$ and $\gamma_{\phi} / \lambda$. (c) The final fidelity $F(T)$ versus $\kappa / \lambda$ and $\gamma / \lambda$. 
(a)

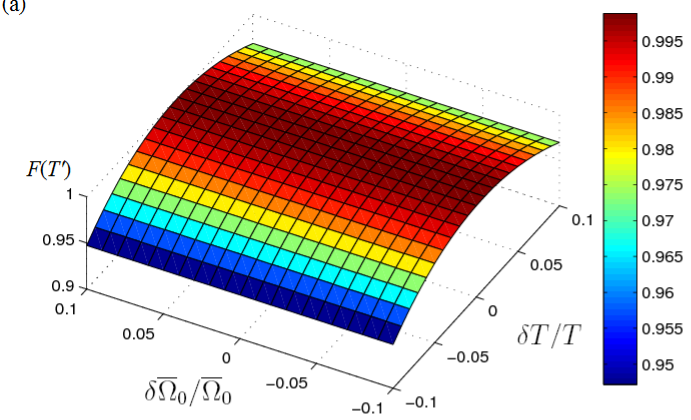

(b)

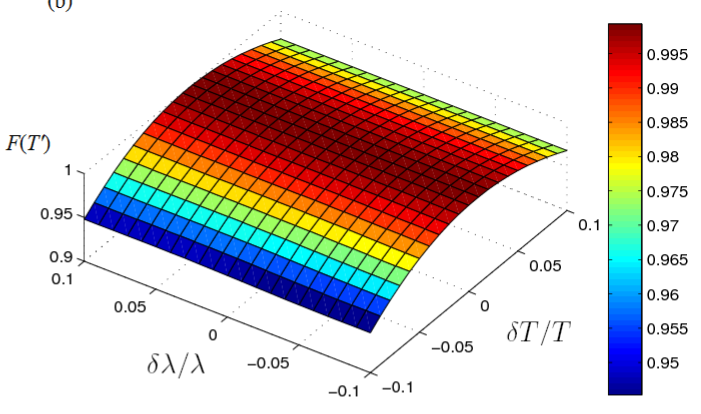

(c)

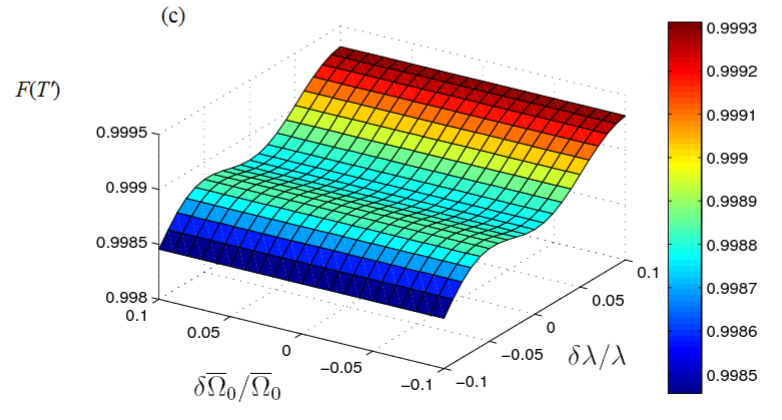

FIG. 7: (a) The fidelity $F\left(T^{\prime}\right)$ versus $\delta T / T$ and $\delta \bar{\Omega}_{0} / \bar{\Omega}_{0}$. (b) The fidelity $F\left(T^{\prime}\right)$ versus $\delta T / T$ and $\delta \lambda / \lambda$. (c) The fidelity $F\left(T^{\prime}\right)$ versus $\delta \bar{\Omega}_{0} / \bar{\Omega}_{0}$ and $\delta \lambda / \lambda$. 